\title{
Assessing Diabetes Distress and Sleep Quality in Young Adults with Type 1 Diabetes Using FreeStyle Libre: A Prospective Cohort Study
}

\author{
Ayman A. Al Hayek (D) - Mohamed A. Al Dawish
}

Received: April 22, 2020 / Published online: June 4, 2020

(c) The Author(s) 2020
Results: Of 95 patients analyzed, significant reductions were observed in mean DDS (3.8 vs. $2.5 ; p<0.001)$ and PSQI (8.7 vs. $3.9 ; p<0.001)$ scores from baseline to 3 months. Furthermore, HbA1c and confirmed hypoglycemia episodes per month also decreased from baseline to 3 months (HbA1c 8.3 vs. $7.7 \%$ [67 vs. $61 \mathrm{mmol} / \mathrm{mol}], p<0.001$; hypoglycemia episodes 3.0 vs. $2.3, p<0.001)$. In contrast, mean frequency of blood glucose testing per day increased from baseline to 3 months ( 2.5 vs. 5.2; $p<0.001)$.

Conclusion: These data demonstrate improvements in diabetes distress and sleep quality as well as glycemic outcomes following 3 months' FSL use in young adults with T1DM.

Keywords: Psychological distress; Psychosocial factors; Sleep; Type 1 diabetes; Wearable electronic devices
Digital Features To view digital features for this article go to https://doi.org/10.6084/m9.figshare.12357866.

A. A. Al Hayek $(\bowtie) \cdot$ M. A. Al Dawish Department of Endocrinology and Diabetes, Diabetes Treatment Center, Prince Sultan Military Medical City, Riyadh, Saudi Arabia e-mail: ayman.alhayek@yahoo.com 


\section{Key Summary Points}

The burden of diabetes, its potential complications, and related self-care activities can induce negative psychosocial effects in patients with T1DM.

This study aimed to investigate the effects of 3 months' use of FSL on diabetes distress and sleep quality in young adults with T1DM in Saudi Arabia. Additional aims were to assess changes in $\mathrm{HbA1c}$, hypoglycemia episodes, and frequency of blood glucose testing.

The data demonstrate improvements in diabetes distress and sleep quality following 3 months' use of FSL in this population. HbA1c and the number of confirmed hypoglycemia episodes per month also decreased. In contrast, the frequency of blood glucose testing increased following 3 months of FSL use.

In populations where psychosocial influences are greater, such as young adults, use of flash glucose monitoring devices has the potential to reduce distress associated with T1DM as well as provide positive glycemic outcomes.

\section{INTRODUCTION}

Type 1 diabetes mellitus (T1DM), or insulindependent diabetes mellitus, is an autoimmune disorder characterized by a relative or absolute deficiency of insulin. The onset of T1DM usually occurs in childhood or adolescence, although the symptoms sometimes develop much later in life [1]. The incidence rate of T1DM has soared in Saudi Arabia over the past 3 decades [2]. A recent report from the International Diabetes Federation showed that Saudi Arabia has the third-highest number of individuals aged $0-19$ years with T1DM in the
Middle East and North Africa region, with 3700 new cases of T1DM diagnosed in 2019 [3].

Living with T1DM is inherently complex and necessitates many self-care activities such as perpetual medication taking, diet control, physical activity, and monitoring of blood glucose [4]. These behaviors are required to maintain glycated hemoglobin (HbA1c) within the target range to prevent the onset of adverse complications [4]. The burdens of self-care and living with the complications associated with diabetes or the possibility of their development can bring about negative psychosocial effects in individuals with T1DM [4].

To ease the burden of self-care and facilitate self-monitoring of glucose, newer technologies have been developed [5]. An example of such a technology is the flash glucose monitoring system (FreeStyle Libre [FSL]; Abbott Diabetes Care, Witney, UK). FSL chemically reads and continuously measures the glucose levels in the interstitial fluid from cells underneath the skin: an ambulatory glucose profile is generated following transfer of the data from the sensor to the reader [5]. Due to factory calibration, this system removes the requirement for frequent finger pricks that are often used in self-monitoring of blood glucose levels [6]. Moreover, continuous glucose monitoring allows glucose levels to be monitored overnight with reduced interruption to sleep because of the lack of an alarm system [7]. Flash glucose monitoring makes self-monitoring of glucose levels more convenient, potentially making it easier for diabetic individuals to comply with such a regimen [8]. Furthermore, increased awareness of their diabetic condition has been observed in patients using flash glucose monitoring because of the ease of glucose monitoring and a graphic depiction of changes in glucose levels over time [9]. FSL is thought to have greater cost-effectiveness than self-monitoring of blood glucose despite greater direct medical costs [10]. Potential disadvantages of FSL include a lack of alarm system to alert the user to low- or high-glucose concentrations as well as a lack of accuracy at low glucose levels, similar to other continuous glucose monitoring systems [11].

Diabetes-related distress, defined as negative emotions (such as frustration, anger, fear, and 
stress) of managing diabetes, is one important psychosocial factor that affects glycemic control in young adults with T1DM [12]. It can stem from feelings of negative social perception, powerlessness, and distress associated with eating [13] as well as from negative interactions with family, friends, and health professionals [12]. Many adolescents with T1DM experience diabetes-related distress, and this condition is often associated with suboptimal glycemic control, low levels of self-care, and reduced selfefficacy [12]. Previous studies of youths with T1DM have shown that chronic diabetes-related distress is associated with higher levels of depressive symptoms and higher HbA1c levels $[13,14]$.

An interesting area to investigate is whether the use of diabetes technologies could help alleviate diabetes-related distress in T1DM individuals. The diabetes distress scale (DDS) was developed to measure emotional distress in T1DM and type 2 diabetes mellitus (T2DM) patients; it demonstrates high reliability and validity [15].

Sleep quality is an important factor that may impact diabetes control [16]. Children and adolescents with T1DM have shorter sleep duration compared with those without diabetes [16]. This is likely due to sleep disturbances unique to T1DM, which include recurrent nocturnal hypoglycemia as well as alterations in sleep architecture caused by rapid fluctuations in glucose levels [17], the need to treat low blood glucose levels overnight [18, 19], and alarms from diabetes monitoring or treatment devices [19]. A recent integrative review of 17 original studies on sleep and T1DM reported that young adults with T1DM have large variations in sleep duration, poor quality of sleep, and sleep deficiency [20]. An improvement in sleep quality has been observed in young children (2-5 years) using continuous glucose monitoring; however, continuous glucose monitoring negatively impacted parents' sleep [21]. However, the impact of newer diabetic technologies on sleep quality in young adults with T1DM has not been extensively studied.

The Pittsburgh Sleep Quality Index (PSQI) was originally developed in 1989 to measure sleep quality for psychiatric research as well as clinical practice [22]. The index has since been used to assess sleep quality in patients suffering from T1DM [18] as well as T2DM [23]. An Arabic version has been developed and tested in a nonclinical setting [24] as well as in patients with coronary artery disease [25].

Previous work has focused on evaluating the effect of flash glucose monitoring on several variables: glycemic control, fear of hypoglycemia and health-related quality of life [5], and treatment satisfaction and mental well-being [26] in adolescents and young adults with T1DM in Saudi Arabia. Although this work has demonstrated the positive effects of FSL on such variables, it is currently unknown whether the use of FSL influences other behavioral, lifestyle, and emotional aspects of diabetes. Therefore, the aim of this study is to investigate changes in diabetes distress and sleep quality associated with 3 months of FSL use in young adults suffering from T1DM and treated with multipledose insulin injection in Saudi Arabia as part of comprehensive diabetic management. To our knowledge, no such study has previously been performed in this population of patients. Additional aims were to assess changes in HbA1c, hypoglycemia episodes, and frequency of blood glucose testing associated with 3 months of FSL use within this population.

\section{METHODS}

\section{Study Design}

A prospective cohort study was conducted in T1DM patients recruited from the Diabetes Treatment Center, Prince Sultan Military Medical City (PSMMC), Riyadh, Saudi Arabia, between March 2019 and October 2019. Patients were sampled using convenience sampling. A sample of 95 T1DM patients aged between 18 and 40 years was achieved. Patients were included if they: used the conventional finger-pricking method for self-testing of glucose, were first-time users of the FSL system, and were receiving insulin treatment via multiple dose injection for at least 6 months prior to study start. Patients were excluded if they: were using another interstitial glucose monitoring 
system concurrently or had used one in the 6 months prior to study start, had a dermatologic disorder or change at the site of sensor application within 6 months of study start, were diagnosed with any depression disorders or severe or unstable medical conditions, severe hypoglycemia (requiring third-party assistance), diabetic ketoacidosis, or a hyperosmolar-hyperglycemic state in the 6 months prior to study start, or were pregnant at study start.

Patients were assessed at a baseline visit and followed up once at 3 months. Patients attended the diabetes treatment center for their baseline and follow-up visits. Appointments were arranged as per the center protocol for follow-up with the treating physician and educator. At the baseline visit, the FSL sensor was attached to the back of the upper arm by a trained diabetes educator, and patients were educated and trained on the proper application of the sensor. All participants were given contact information of the diabetes educator, who could be accessed at any point of time during this study.

\section{Sample Size}

Sample size was calculated using Statulator, an online sample size calculator [27]. Based on detecting a difference of 1 point in the test scores between baseline and 3 months (paired samples) with 95\% confidence and 80\% power, and assuming a standard deviation (SD) of 3 in differences between baseline and 3 months, a sample size of at least 73 patients was needed. A final sample size of 95 patients was achieved.

\section{Variables}

A trained interviewer provided the patient with the two questionnaires to be completed at baseline and the 3-month follow-up visit: the Arabic versions of the DDS [28] and the PSQI [24]. Both questionnaires pose a number of close-ended questions. The Arabic version of the PSQI is a 19-item self-administered questionnaire evaluating quality of sleep [24]. The 19 items comprise 7 factors: subjective sleep quality, sleep latency, sleep duration, habitual sleep efficiency, sleep disturbances, use of sleep medications, and daytime dysfunction. A global score is generated by the addition of component scores and ranges from 0 to 21 , with a higher score demonstrating poorer sleep quality. The PSQI takes, on average, $8 \mathrm{~min}$ to complete. The DDS17 is a 17-item questionnaire examining distress experiences among patients with diabetes. Each individual item is measured on a scale of 1 (no distress) to 6 (serious distress), and an average composite score is also determined. The DDS17 is composed of four subscales: emotional distress (EB), regimen distress (RD), interpersonal distress (ID), and physician distress (PD) [15]. An Arabic version of the DDS has been developed, which has been shown to be a valid and reliable tool to measure diabetes-related distress in diabetes patients, demonstrating cross-cultural adaptation of the DDS [28]. The DDS takes approximately 5-8 min to complete.

For the DDS, scores were generated for the four components (emotional burden, physician-related distress, regimen-related distress, and interpersonal distress) as well as an overall score. For the PSQI, scores were generated for the seven components (subjective sleep quality, sleep latency, sleep duration, sleep efficiency, sleep disturbance, use of sleep medication, and daytime dysfunction) as well as an overall score.

Patients' demographic data (age, gender, weight, and height), clinical characteristics (duration of T1DM, HbA1c, number of confirmed hypoglycemia episodes per month, and frequency of blood glucose testing), and treatment history were collected at baseline using a standardized case record form at the baseline visit. Data were sourced from electronic medical records. HbA1c, number of confirmed hypoglycemia episodes, and frequency of blood glucose testing were collected in a similar manner at the 3-month follow-up visit. HbA1c was measured using the COBAS INTEGRA 400 plus/ 800 analyzers at the central laboratory of PSMMC. Confirmed hypoglycemia was defined as blood glucose $\leq 70 \mathrm{mg} / \mathrm{dl}$. Frequency of blood glucose testing was defined as the average number of blood glucose tests using finger-prick per day at baseline and the average FSL scanning frequency per day at 3 months. A body 
mass index (BMI, $\mathrm{kg} / \mathrm{m}^{2}$ ) variable was generated for each patient based on weight and height measurements at baseline. Categorical variables were generated for BMI as follows: normal $\left(<25 \mathrm{~kg} / \mathrm{m}^{2}\right)$, overweight $\left(25.0-29.9 \mathrm{~kg} / \mathrm{m}^{2}\right)$, and obese $\left(\geq 30 \mathrm{~kg} / \mathrm{m}^{2}\right)$.

Participants were then shown how to confirm their blood glucose levels using a capillary measurement and the in-built blood glucose meter reader in the event of imminent and/or suspected hypoglycemia, rapidly changing glucose levels, or when their symptoms failed to match the reading on the system. Data were then collected from the blood glucose meter readers, computed to determine the total number of capillary tests conducted and the frequency of hypoglycemia during the followup study period, and subsequently entered into the patient records. When the study was completed, all data from the sensors were computed and the corresponding ambulatory glucose profiles generated to determine the total number of scans performed during the 90-day study period.

\section{Analysis}

Descriptive statistics, including measures of central tendency and dispersion for continuous variables (mean, SD, median, interquartile range [IQR], and percentiles) and frequencies with proportions for categorical variables, were used. Distribution of the data was assessed for normality using the Q-Q plot and the ShapiroWilk test. Differences between baseline and 3-month variables were assessed using the paired t-test (if normally distributed) or the Wilcoxon matched-pairs signed-rank test (if non-normally distributed). Missing data are presented as such, without imputation. All statistical analyses were performed using StataIC (StataCorp, version 16). For data interpretation, the mean number of scans per day was considered.

\section{Ethics}

Participants or their caregivers gave written informed consent to participate in the study.

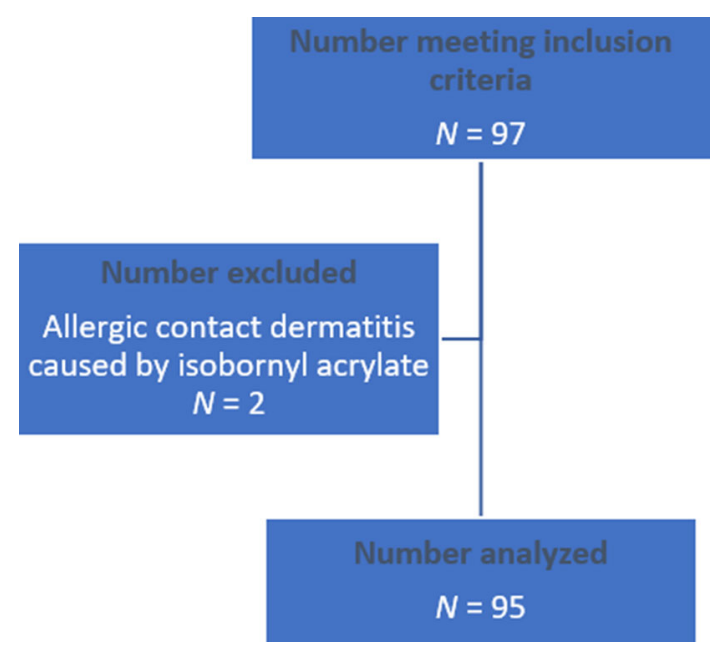

Fig. 1 Study flow chart

The study protocol was approved by the Research Ethics Committee of the PSMMC in accordance with the Helsinki Declaration of 1964, revised in 2013 (ethical approval no. 1200-HP-01-R079). All participants could withdraw at any point without reason or prior notice.

\section{RESULTS}

\section{Cohort Demographics}

A total of 97 patients met the inclusion criteria for the study. However, two of these patients experienced allergic contact dermatitis caused by isobornyl acrylate, part of the adhesive component of the FSL sensor, and were therefore excluded from the study. No patients were lost to follow-up. Analysis was performed on the remaining 95 patients, as shown in Fig. 1. Baseline characteristics are shown in Table 1.

\section{Diabetes Distress}

DDS significantly decreased from baseline (mean score 3.8 ) to 3 months (mean score 2.5; $p<0.001$ ), demonstrating a reduction in distress associated with diabetes. All four components of the DDS score decreased over the 3 months (Table 2). 
Table 1 Baseline characteristics

\begin{tabular}{|c|c|}
\hline & $(N=95)$ \\
\hline \multicolumn{2}{|l|}{ Age (years) } \\
\hline Mean (SD) & $20.9(2.2)$ \\
\hline Median (IQR) & $20(19,23)$ \\
\hline \multicolumn{2}{|l|}{ Gender } \\
\hline Female, $n(\%)$ & $42(44.2)$ \\
\hline Male, $n(\%)$ & $53(55.8)$ \\
\hline \multicolumn{2}{|l|}{$\operatorname{BMI}\left(\mathrm{kg} / \mathrm{m}^{2}\right)$} \\
\hline Normal (18.5-24.9), $n(\%)$ & $19(20.0)$ \\
\hline Overweight (25-29.9), $n$ (\%) & $59(62.1)$ \\
\hline Obese $(\geq 30), n(\%)$ & $17(17.9)$ \\
\hline \multicolumn{2}{|l|}{ Diabetes duration (years) } \\
\hline Mean (SD) & $7.2(2.9)$ \\
\hline Median (IQR) & $7(5,8)$ \\
\hline \multicolumn{2}{|c|}{ Average blood glucose testing using finger-prick (tests/day) } \\
\hline Mean (SD) & $2.5(0.9)$ \\
\hline Median (IQR) & $2(2,3)$ \\
\hline \multicolumn{2}{|c|}{ Confirmed hypoglycemia episodes (number/month) } \\
\hline Mean (SD) & $3.0(1.5)$ \\
\hline Median (IQR) & $3(2,4)$ \\
\hline \multicolumn{2}{|l|}{ HbAlc } \\
\hline Mean (SD), \% & $8.3(1.0)$ \\
\hline Mean (SD), $\mathrm{mmol} / \mathrm{mol}$ & $67(10.9)$ \\
\hline$\leq 7 \%(\leq 53 \mathrm{mmol} / \mathrm{mol}), n(\%)$ & $7(7.4)$ \\
\hline $\begin{array}{l}>7 \text { and } \leq 9 \%(>53 \\
\text { and } \leq 75 \mathrm{mmol} / \mathrm{mol}), n(\%)\end{array}$ & $67(70.5)$ \\
\hline$>9 \%(>75 \mathrm{mmol} / \mathrm{mol}), n(\%)$ & $21(22.1)$ \\
\hline
\end{tabular}

$I Q R$ interquartile range, $S D$ standard deviation

\section{Sleep Quality}

PSQI significantly decreased from baseline (mean score 8.7) to 3 months (mean score 3.9; $p<0.001$ ), demonstrating an improvement in sleep quality. PSQI component scores are shown in Table 3.

\section{HbA1c, Hypoglycemia Episodes, and Frequency of Blood Glucose Testing}

Mean HbA1c and the number of individuals with poor control decreased from baseline to 3 months (Table 4). Furthermore, the mean number of confirmed hypoglycemia episodes decreased, and fewer patients experienced five or more episodes per month, demonstrating an improvement in glycemic control (Table 4). The mean frequency of blood glucose testing increased, with more patients undertaking five or more blood glucose tests per day following FSL use (Table 4).

\section{DISCUSSION}

The results of this study demonstrate a significant reduction in both DDS and PSQI scores following 3 months of FSL use in this population of T1DM patients, indicative of a reduction in patient distress and an improvement in sleep quality, respectively. Furthermore, HbA1c and the number of confirmed hypoglycemia episodes per month decreased following 3 months of FSL use, demonstrating greater diabetes control in this set of patients. In contrast, the frequency of blood glucose testing increased following 3 months of FSL use.

Previous studies have examined the effects of FSL on DDS scores. One such study, which investigated 25 adult patients (mean age 39.8 years) attending a T1DM clinic in the UK, demonstrated a significant reduction in DDS scores following 16 weeks of FSL use [29]. Furthermore, an observational study of 900 patients (median age 42 years) with T1DM at two UK hospitals showed an improvement in overall DDS score in $90 \%$ of patients following FSL use (median follow-up 245 days) [30]. However, a multicenter randomized control trial (RCT) of 241 adult patients ( $\geq 18$ years) with well-controlled diabetes (HbA1c $\leq 7.5 \%$; $\leq 58 \mathrm{mmol} / \mathrm{mol})$ from 23 diabetes centers across Europe showed no difference in DDS ( $p=0.7634)$ following 6 months of FSL use [31]. Such studies have focused on adult T1DM patients and European populations. A prospective study in Saudi Arabia demonstrated 
Table 2 Diabetes Distress Scale (DDS) scores

\begin{tabular}{|c|c|c|c|}
\hline \multirow[t]{2}{*}{ DDS scores } & \multicolumn{2}{|c|}{ Mean (SD) } & \multirow{2}{*}{$\begin{array}{l}\text { Wilcoxon matched-pairs } \\
\text { signed-rank test }\end{array}$} \\
\hline & Baseline & 3 months & \\
\hline Emotional burden & $3.9(0.7)$ & $2.5(0.5)$ & $<0.001$ \\
\hline Physician-related distress & $3.3(0.7)$ & $2.4(0.7)$ & $<0.001$ \\
\hline Regimen-related distress & $4.3(0.6)$ & $2.5(0.5)$ & $<0.001$ \\
\hline Interpersonal distress & $3.4(0.9)$ & $2.6(0.7)$ & $<0.001$ \\
\hline Total DDS score & $3.8(0.4)$ & $2.5(0.3)$ & $<0.001$ \\
\hline
\end{tabular}

$S D$ standard deviation

Table 3 Pittsburgh Sleep Quality Index (PSQI) scores

\begin{tabular}{llll}
\hline PSQI scores & Mean (SD) & & $\begin{array}{c}\text { Wilcoxon matched-pairs } \\
\text { signed-rank test }\end{array}$ \\
\cline { 2 - 3 } & Baseline & 3 months & $<0.001$ \\
\hline Component 1-subjective sleep quality & $1.8(0.9)$ & $0.3(0.7)$ & $<0.001$ \\
Component 2-sleep latency & $2.1(0.5)$ & $1.3(0.5)$ & $<0.001$ \\
Component 3-sleep duration & $1.3(0.9)$ & $0.3(0.5)$ & $<0.001$ \\
Component 4-sleep efficiency & $1.2(1.2)$ & $0.2(0.6)$ & 1.000 \\
Component 5-sleep disturbance & $1.0(0.1)$ & $1.0(0.0)$ & 0.0037 \\
Component 6-use of sleep medication & $0.5(0.7)$ & $0.7(0.6)$ & $<0.001$ \\
Component 7-daytime dysfunction & $0.8(0.6)$ & $0.1(0.3)$ & $<0.001$ \\
Global PSQI score & $8.7(2.5)$ & $3.9(1.5)$ &
\end{tabular}

$S D$ standard deviation

a decrease in total DDS score in 187 children and adolescents aged 13-19 years with T1D following 12 weeks of flash glucose monitoring (baseline 2.93 vs. 12 weeks $2.46 ; p=0.001$ ) [32]. A recent study reported that continuous glucose monitoring in adolescents with T1DM is associated with lower diabetes-related distress [33]. Additionally, structured educational programs can aid patients in their understanding and use of FSL and its available glycemic information to optimize their treatment; one such program (FLASH) reported lower DDS scores after 6 months compared with patients who did not participate in the program $(p=0.029)$ [34]. This indicates that such programs can positively impact monitoring and treatment of T1DM.
To our knowledge, no previous studies have examined the effects of FSL on PSQI scores. A study that is currently being conducted in New Zealand aims to assess the impact of flash glucose monitoring on PSQI following 6 months of FSL use in patients recruited from diabetes clinics (WHO International Clinical Trials Registry no. U1111-1205-5784) [35]. An online survey of T1DM patients using continuous glucose monitoring reported some patients "sleeping more easily" with the use of such devices, whereas others reported sleep disruptions due to sensor alarms [36].

The impact of FSL on HbA1c and hypoglycemia has been assessed in several RCTs. One study, conducted in 241 ( $\geq 18$ years) T1DM 
Table 4 HbAlc, confirmed hypoglycemia episodes, and frequency of blood glucose testing

\begin{tabular}{|c|c|c|c|}
\hline & Baseline & 3 months & $\begin{array}{l}\text { Wilcoxon matched-pairs } \\
\text { signed-rank test }\end{array}$ \\
\hline \multicolumn{4}{|l|}{ HbAlc, $n(\%)$} \\
\hline$\leq 7 \%(\leq 53 \mathrm{mmol} / \mathrm{mol})$ & $7(7.4)$ & $27(28.4)$ & \\
\hline$>7$ and $\leq 9 \%(>53$ and $\leq 75 \mathrm{mmol} / \mathrm{mol})$ & $67(70.5)$ & $58(61.0)$ & \\
\hline$>9 \%(>75 \mathrm{mmol} / \mathrm{mol})$ & $21(22.1)$ & $10(10.5)$ & \\
\hline Mean (SD), \% & $8.3(1.0)$ & $7.7(0.9)$ & $<0.001$ \\
\hline Mean (SD), $\mathrm{mmol} / \mathrm{mol}$ & $67(10.9)$ & $61(9.8)$ & \\
\hline \multicolumn{4}{|c|}{ Confirmed hypoglycemia episodes (number/month), $n$ (\%) } \\
\hline$\leq 1$ & $15(15.8)$ & $23(24.1)$ & \\
\hline $2-4$ & $62(65.3)$ & $69(72.6)$ & \\
\hline$\geq 5$ & $18(19.0)$ & $3(3.2)$ & \\
\hline Mean (SD) & $3.0(1.5)$ & $2.3(1.1)$ & $<0.001$ \\
\hline \multicolumn{4}{|c|}{ Frequency of blood glucose testing per day*, mean (SD) } \\
\hline$\leq 1$ & $9(9.5)$ & $0(0.0)$ & \\
\hline $2-4$ & $85(89.5)$ & $27(28.4)$ & \\
\hline$\geq 5$ & $1(1.0)$ & $68(71.6)$ & \\
\hline Mean (SD) & $2.5(0.9)$ & $5.2(1.2)$ & $<0.001$ \\
\hline
\end{tabular}

patients using FSL across 23 European diabetes centers demonstrated a $38 \%$ reduction in time spent in hypoglycemia at 6 months versus baseline, but found no change in baseline HbA1c of $6.7 \%$ [31]. Another RCT, conducted in 20 patients with impaired hypoglycemia awareness or severe hypoglycemia at a single site in the UK, showed no significant changes in time spent in hypoglycemia or in HbA1c following 8 weeks of FSL [37]. An observational study of 25 adult patients (mean age 39.8 years) attending diabetes clinics in the UK demonstrated a reduction in mean $\mathrm{HbA} 1 \mathrm{c}$ from 8.0 to $7.5 \% \quad(64-58 \mathrm{mmol} / \mathrm{mol} ; \quad-0.48 \%, p=0.001)$ following 16 weeks of FSL [29]. The same study demonstrated a reduction in the frequency of hypoglycemia episodes at weeks 1-2 of FSL use (17 episodes) compared with weeks 15-16 (12 episodes; $p=0.019$ ) [29]. Furthermore, an observational study of patients using FSL at a single site in the UK reported a median $\mathrm{HbA} 1 \mathrm{c}$ reduction of $0.2 \% \quad(\mathrm{IQR}-0.7$ to $0.3 \%)$ [2.5 $\mathrm{mmol} / \mathrm{mol} \quad(-8.0$ to $2.75 \mathrm{mmol} / \mathrm{mol})]$ between $2014 / 2015$ to 2016 [38].

Frequency of flash glucose monitoring scanning has been shown to be associated with improved glycemic control, with lower HbA1c $(8.0 \%$ [64 mmol/mol] in patients scanning the least [4.4 scans/day] vs. $6.7 \%$ [50 mmol/mol] in patients scanning the most [48.1 scans/day]; $p<0.001)$ and reduced time spent in hyperglycemia or hypoglycemia [39].

Limitations to this study include the use of convenience sampling as well as the lack of a control group. A random sample of patients would be preferable, as would the inclusion of an appropriate control group to evaluate changes in the same parameters in the control group. 
The study may also be subject to selection bias because of the recruitment of patients by their doctors. Furthermore, there is the possibility of recall bias, as with any observational study using patient questionnaires. The exclusion of patients with a history of severe hypoglycemia is a further limitation as it would be of great interest to study the effects of FSL on diabetes distress and sleep quality in this group of patients. The comparison made between frequency of finger pricks at baseline versus frequency of FSL scanning at 3 months as measurements of frequency of glucose monitoring also has its limitations; however, frequency of FSL scanning is able to provide some indication of the level of glucose monitoring. Future studies should aim to determine any longer-term benefits of FSL in terms of diabetes distress and sleep quality. Larger sample sizes and random sampling would be required in future studies.

\section{CONCLUSIONS}

Our data demonstrate a significant reduction in diabetes distress, sleep quality, HbA1c, and confirmed hypoglycemia episodes following 3 months' use of FSL in a population of young adult T1DM patients in Saudi Arabia. In such a population, where psychosocial influences may be greater than in older adults, the use of flash glucose monitoring devices has the potential to reduce the burden of diabetes and improve psychosocial wellbeing. This study contributes additional data to the field and demonstrates the benefits of such technologies in diabetes.

\section{ACKNOWLEDGEMENTS}

We thank the participants of the study.

Funding. No funding or sponsorship was received for conducting this study. The rapid service fee and open access fee were funded by Abbott Diabetes Care.

Authorship. Both named authors meet the International Committee of Medical Journal
Editors criteria for authorship for this article, take responsibility for the integrity of the work as a whole, and have given their approval for this version to be published.

Authorship Contributions. Ayman A. Al Hayek and Mohamed A. Al Dawish were involved in acquisition and interpretation of the data; they reviewed the manuscript and contributed to the discussion.

Medical Writing Assistance. Medical writing assistance in the preparation of this article was provided by Dr. Abigail Holland of Connect Communications. Support for this assistance was funded by Abbott Diabetes Care.

Disclosures. Ayman A. Al Hayek and Mohamed A. Al Dawish have nothing to disclose.

Compliance with Ethics Guidelines. The study protocol was approved by the Research Ethics Committee of the PSMMC in accordance with the Helsinki Declaration of 1964, revised in 2013 (ethics approval no. HP-01-R079/project no. 1200). Participants or their parents/caregivers were advised of their roles in this study, and their signed informed consent was obtained prior to recruitment of the subjects. All participants reserved an unconditional or absolute right of withdrawal at any point of time in the study without giving any reason or prior notice. Consent for data publication was obtained from parents/caregivers.

Data Availability. The datasets generated during and/or analyzed during the current study are available from the corresponding author on reasonable request.

Open Access. This article is licensed under a Creative Commons Attribution-NonCommercial 4.0 International License, which permits any non-commercial use, sharing, adaptation, distribution and reproduction in any medium or format, as long as you give appropriate credit to the original author(s) and the source, provide a link to the Creative Commons licence, and indicate if changes were made. The images or 
other third party material in this article are included in the article's Creative Commons licence, unless indicated otherwise in a credit line to the material. If material is not included in the article's Creative Commons licence and your intended use is not permitted by statutory regulation or exceeds the permitted use, you will need to obtain permission directly from the copyright holder. To view a copy of this licence, visit http://creativecommons.org/licenses/by$\mathrm{nc} / 4.0 /$.

\section{REFERENCES}

1. Harjutsalo V, Lammi N, Karvonen M, Groop P-H. Age at onset of type 1 diabetes in parents and recurrence risk in offspring. Diabetes. 2010;59(1): 210-4.

2. Robert AA, Al-Dawish A, Mujammami M, Dawish MAA. Type 1 diabetes mellitus in Saudi Arabia: a soaring epidemic. Int J Pediatr. 2018. https://doi. org/10.1155/2018/9408370.

3. Federation ID. Saudi Arabia Country report 20102045. 2019. Available from: https://diabetesatlas. org/data/en/country/174/sa.html. Accessed 6 Jan 2020

4. Dennick K, Sturt J, Speight J. What is diabetes distress and how can we measure it? A narrative review and conceptual model. J Diabetes Complicat. 2017;31(5):898-911. https://doi.org/10.1016/j. jdiacomp.2016.12.018.

5. Al Hayek AA, Robert AA, Al Dawish MA. Evaluation of FreeStyle Libre flash glucose monitoring system on glycemic control, health-related quality of life, and fear of hypoglycemia in patients with type 1 diabetes. Clin Med Insights Endocrinol Diabetes. 2017;10:1179551417746957. https://doi.org/10. $1177 / 1179551417746957$.

6. Hoss U, Budiman ES. Factory-calibrated continuous glucose sensors: the science behind the technology. Diabetes Technol Ther. 2017;19(S2):S44-50.

7. Adolfsson P, Rentoul D, Klinkenbijl B, Parkin CG. Hypoglycaemia remains the key obstacle to optimal glycaemic control-continuous glucose monitoring is the solution. Eur Endocrinol. 2018;14(2):50-6.

8. Slattery D, Choudhary P. Clinical use of continuous glucose monitoring in adults with type 1 diabetes. Diabetes Technol Ther. 2017;19(S2):S55-61.
9. Danesi G, Pralong M, Panese F, Burnand B, Grossen M. Techno-social reconfigurations in diabetes (self-) care. Soc Stud Sci. 2020. https://doi.org/10.1177/ 0306312720903493.

10. Bilir SP, Hellmund R, Wehler B, Li H, Munakata J, Lamotte M. Cost-effectiveness analysis of a flash glucose monitoring system for patients with type 1 diabetes receiving intensive insulin treatment in Sweden. Eur Endocrinol. 2018;14(2):73-9.

11. Blum A. Freestyle libre glucose monitoring system. Clin Diabetes. 2018;36(2):203-4.

12. Hagger V, Hendrieckx C, Sturt J, Skinner TC, Speight J. Diabetes distress among adolescents with type 1 diabetes: a systematic review. Curr Diab Rep. 2016;16(1):9. https://doi.org/10.1007/s11892-0150694-2.

13. Powers MA, Richter SA, Ackard DM, Craft C. Diabetes distress among persons with type 1 diabetes. Diabetes Educ. 2017;43(1):105-13. https://doi.org/ $10.1177 / 0145721716680888$.

14. Iturralde E, Rausch JR, Weissberg-Benchell J, Hood KK. Diabetes-related emotional distress over time. Pediatrics. 2019. https://doi.org/10.1542/peds. 2018-3011.

15. Polonsky WH, Fisher L, Earles J, et al. Assessing psychosocial distress in diabetes: development of the diabetes distress scale. Diabetes Care. 2005;28(3):626-31. https://doi.org/10.2337/ diacare.28.3.626.

16. Reutrakul S, Thakkinstian A, Anothaisintawee $\mathrm{T}$, et al. Sleep characteristics in type 1 diabetes and associations with glycemic control: systematic review and meta-analysis. Sleep Med. 2016;23: 26-45. https://doi.org/10.1016/j.sleep.2016.03.019.

17. Pillar G, Schuscheim G, Weiss R, et al. Interactions between hypoglycemia and sleep architecture in children with type 1 diabetes mellitus. J Pediatr. 2003;142(2):163-8. https://doi.org/10.1067/mpd. 2003.66 .

18. Patel NJ, Savin KL, Kahanda SN, et al. Sleep habits in adolescents with type 1 diabetes: variability in sleep duration linked with glycemic control. Pediatr Diabetes. 2018. https://doi.org/10.1111/pedi. 12689.

19. Bergner EM, Williams R, Hamburger ER, et al. Sleep in teens with type 1 diabetes: perspectives from adolescents and their caregivers. Diabetes Educ. 2018;44(6):541-8. https://doi.org/10.1177/ 0145721718799086.

20. Griggs S, Redeker NS, Grey M. Sleep characteristics in young adults with type 1 diabetes. Diabetes Res 
Clin Pract. 2019;150:17-26. https://doi.org/10. 1016/j.diabres.2019.02.012.

21. Sinisterra M, Hamburger S, Tully C, Hamburger E, Jaser S, Streisand R. Young children with type 1 diabetes: sleep, health-related quality of life, and continuous glucose monitor use. Diabetes Technol Ther. 2020. https://doi.org/10.1089/dia.2019.0437.

22. Buysse DJ, Reynolds CF 3rd, Monk TH, Berman SR, Kupfer DJ. The Pittsburgh Sleep Quality Index: a new instrument for psychiatric practice and research. Psychiatry Res. 1989;28(2):193-21313. https://doi.org/10.1016/0165-1781(89)90047-4.

23. Telford O, Diamantidis CJ, Bosworth HB, et al. The relationship between Pittsburgh Sleep Quality Index subscales and diabetes control. Chronic Illn. 2019;15(3):210-9. 1742395318759587.

24. Suleiman KH, Yates BC, Berger AM, Pozehl B, Meza J. Translating the pittsburgh sleep quality index into Arabic. West J Nurs Res. 2010;32(2):250-68. https://doi.org/10.1177/0193945909348230.

25. Suleiman KH, Al-Hadid L, Duhni A. Psychometric testing of the Arabic version of the Pittsburgh Sleep Quality Index (A-PSQI) among coronary artery disease patients in Jordan. J Nat Sci Res. 2012;2(8): $15-9$.

26. Al Hayek AA, Al Dawish MA. The potential impact of the FreeStyle Libre flash glucose monitoring system on mental well-being and treatment satisfaction in patients with type 1 diabetes: a prospective study. Diabetes Ther. 2019;10(4):1239-48. https://doi.org/10.1007/s13300-019-0616-4.

27. Dhand NK, Khatkar MS. Statulator: An online statistical calculator. Sample Size Calculator for Comparing Two Paired Means. 2014. Accessed at: https://statulator.com/SampleSize/ss2PM.html. Accessed 18 May 2020

28. Darawad MW, Hammad S, Samarkandi OA, Hamdan-Mansour AM, Khalil AA. Evaluating the psychometric properties of the Arabic version of the Diabetes Distress Scale. J Psychosoc Nurs Ment Health Serv. 2017;55(9):43-51. https://doi.org/10. 3928/02793695-20170818-12.

29. Dover AR, Stimson RH, Zammitt NN, Gibb FW. Flash Glucose Monitoring improves outcomes in a type 1 diabetes clinic. J Diabetes Sci Technol. 2017;11(2):442-3. 1932296816661560 .

30. Tyndall V, Stimson RH, Zammitt NN, et al. Marked improvement in HbA1c following commencement of flash glucose monitoring in people with type 1 diabetes. Diabetologia. 2019;62(8):1349-56. https://doi.org/10.1007/s00125-019-4894-1.

31. Bolinder J, Antuna R, Geelhoed-Duijvestijn P, Kroger J, Weitgasser R. Novel glucose-sensing technology and hypoglycaemia in type 1 diabetes: a multicentre, non-masked, randomised controlled trial. Lancet. 2016;388(10057):2254-63. https://doi. org/10.1016/S0140-6736(16)31535-5.

32. Al Hayek AA, Robert AA, Al Dawish MA. Effectiveness of the freestyle libre flash glucose monitoring system on diabetes distress among individuals with type 1 diabetes: a prospective study. Diabetes Ther. 2020. https://doi.org/10.1007/s13300-020-00793-2.

33. Vesco AT, Jedraszko AM, Garza KP, WeissbergBenchell J. Continuous glucose monitoring associated with less diabetes-specific emotional distress and lower A1c among adolescents with type 1 diabetes. J Diabetes Sci Technol. 2018;12(4):792-9. https://doi.org/10.1177/1932296818766381.

34. Hermanns N, Ehrmann D, Schipfer M, Kroger J, Haak T, Kulzer B. The impact of a structured education and treatment programme (FLASH) for people with diabetes using a flash sensor-based glucose monitoring system: results of a randomized controlled trial. Diabetes Res Clin Pract. 2019;150: 111-21. https://doi.org/10.1016/j.diabres.2019.03. 003.

35. Boucher SE, Gray AR, de Bock M, et al. Effect of 6 months' flash glucose monitoring in adolescents and young adults with type 1 diabetes and suboptimal glycaemic control: managing diabetes in a 'flash' randomised controlled trial protocol. BMC Endocr Disord. 2019;19(1):50. https://doi.org/10. 1186/s12902-019-0378-z.

36. Pickup JC, Ford Holloway M, Samsi K. Real-time continuous glucose monitoring in type 1 diabetes: a qualitative framework analysis of patient narratives. Diabetes Care. 2015;38(4):544-50. https://doi.org/ 10.2337/dc14-1855.

37. Reddy M, Jugnee N, El Laboudi A, Spanudakis E, Anantharaja S, Oliver N. A randomized controlled pilot study of continuous glucose monitoring and flash glucose monitoring in people with Type 1 diabetes and impaired awareness of hypoglycaemia. Diabet Med. 2018;35(4):483-90. https://doi.org/10. 1111/dme.13561.

38. McKnight JA, Gibb FW. Flash Glucose Monitoring is associated with improved glycaemic control but use is largely limited to more affluent people in a UK diabetes centre. Diabet Med. 2017;34(5):732. https://doi.org/10.1111/dme.13315. 
39. Dunn TC, Xu Y, Hayter G, Ajjan RA. Real-world flash glucose monitoring patterns and associations between self-monitoring frequency and glycaemic measures: a European analysis of over 60 million glucose tests. Diabetes Res Clin Pract. 2018;137:

37-46. https://doi.org/10.1016/j.diabres.2017.12. 015. 